\title{
ALIQUOT SEQUENCE 3630 ENDS AFTER REACHING 100 DIGITS
}

\author{
MANUEL BENITO, WOLFGANG CREYAUFMÜLLER, JUAN L. VARONA, AND PAUL \\ ZIMMERMANN
}

\begin{abstract}
In this paper we present a new computational record: the aliquot sequence starting at 3630 converges to 1 after reaching a hundred decimal digits. Also, we show the current status of all the aliquot sequences starting with a number under 10000; we have reached at leat 95 digits for all of them. In particular, we have reached at least 112 digits for the so-called "Lehmer five sequences", and 101 digits for the "Godwin twelve sequences". Finally, we give a summary showing the number of aliquot sequences of unknown end starting with a number $\leq 10^{6}$.
\end{abstract}

\section{INTRODUCTION}

For a positive integer $n$, let $\sigma(n)$ denote the sum of its divisors (including 1 and $n$ ), and $s(n)=\sigma(n)-n$ the sum of its proper divisors (without $n$ ). A perfect number is a number $n$ such that $s(n)=n$, and an amicable pair of numbers $(n, m)$ satisfies $s(n)=m, s(m)=n$. In a similar way, tuples of numbers $\left(a_{1}, a_{2}, \ldots, a_{l}\right)$ such that $s\left(a_{i}\right)=a_{i+1}$ for $1 \leq i \leq l-1$ and $s\left(a_{l}\right)=a_{1}$ are known as aliquot cycles or sociable numbers.

Given $n$, the way to compute $\sigma(n)$ (and then, $s(n)$ ) is as follows. We find the prime decomposition of $n=p_{1}^{a_{1}} \cdots p_{d}^{a_{d}}$. Then

$$
\begin{aligned}
\sigma\left(p_{1}^{a_{1}} \cdots p_{d}^{a_{d}}\right) & =\left(1+p_{1}+\cdots+p_{1}^{a_{1}}\right) \cdots\left(1+p_{d}+\cdots+p_{d}^{a_{d}}\right) \\
& =\frac{p_{1}^{a_{1}+1}-1}{p_{1}-1} \cdots \frac{p_{d}^{a_{d}+1}-1}{p_{d}-1} .
\end{aligned}
$$

Indeed, if we expand de expression on the right in (1), all the divisors of $p_{1}^{a_{1}} \cdots p_{d}^{a_{d}}$ appear as summands.

By iterating the function $s$, i.e., taking $s^{0}(n)=n$ and $s^{k+1}(n)=s\left(s^{k}(n)\right)$, it appears the so-called aliquot sequence $\left\{s^{k}(n)\right\}_{k=0}^{\infty}$. For each one of these sequences, there are four possibilities:

(i) it terminates at 1 (the previous term being a prime number);

(ii) it reaches a perfect number;

(iii) it reaches an amicable pair or a cycle;

(iv) it is unbounded.

2000 Mathematics Subject Classification. Primary 11Y55; Secondary 11A25.

Key words and phrases. Aliquot sequences, sum of divisors, perfect number, amicable pair, sociable numbers, aliquot cycles.

THIS PAPER HAS BEEN PUBLISHED IN Experimental Mathematics 11 (2002), no. 2, 201206. 
The Catalan-Dickson conjecture says that (iv) does not actually happen. But other researchers disagree with this conjecture and think that there are unbounded sequences; in fact, the alternative conjecture from Guy-Selfridge Guy and Selfridge 75 (see also [Erdös 76]) states that there are many sequences that go to infinity, perhaps almost all those that start at a even number (i.e., the proportion of even integers $n$ such that $\left\{s^{k}(n)\right\}_{k=0}^{\infty}$ is bounded tends to zero).

This new conjecture is based upon the existence of several multiplicative patterns $2^{a} p_{1} \cdots p_{m}$ that, when appearing in the factor decomposition of $n$, they show up again, with a high order of probability, in the factor decomposition of $s(n)$. Following Guy and Selfridge 75], these patterns are called drivers or guides.

By definition, a guide is of the form $2^{a}$, together with a subset of prime factors of $\sigma\left(2^{a}\right)\left(=2^{a+1}-1\right)$. And a driver is of the form $2^{a} v$ with $a>0, v \mid \sigma\left(2^{a}\right)$ and $2^{a-1} \mid$ $\sigma(v)$. This last condition ensures that the power of the prime 2 tends to persist, at least, as much as if the driver is 2, for which the condition is trivial. Actually, drivers are much more stable than guides. As stated in Guy and Selfridge 75, the only drivers are $2,2^{3} \cdot 3,2^{3} \cdot 3 \cdot 5,2^{5} \cdot 3 \cdot 7,2^{9} \cdot 3 \cdot 11 \cdot 31$, and the even perfect numbers. Examples of guides that are not drivers are $2^{a}(a>1), 2^{3} \cdot 5,2^{5} \cdot 3,2^{5} \cdot 3^{2}$, $2^{5} \cdot 3^{2} \cdot 7,2^{7} \cdot 3 \cdot 5, \ldots$

Using (1), it is easy to observe the behavior of drivers (and guides). For instance, let us take $n=2^{3} \cdot 3 \cdot 5 p q$, with $p$ and $q$ prime numbers, $p \neq q, p, q>5$. The same happens if we have more than two prime factors greater than 5 but, by simplicity, let us see this example. We can check that $s(n)=(1+2+4+8) \cdot 4 \cdot 6(p+1)(q+1)-2^{3}$. $3 \cdot 5 p q=15 \cdot 2^{2} \cdot 2 \cdot 3(p+1)(q+1)-2^{3} \cdot 3 \cdot 5 p q=2^{3} \cdot 3 \cdot 5 \cdot[3(p+1)(q+1)-p q]=2^{3} \cdot 3 \cdot 5 m$, with $m$ an odd number that is not divisible by 3 . Then, we get again $2^{3} \cdot 3 \cdot 5$ with 2 and 3 raised to the same power as before (however, it is possible that the power of 5 changes; this may cause the loss of the driver at a later point). The behavior is similar if the prime factors greater than 5 are raised to an odd power, because $1+p+\cdots+p^{a}$ is even when $a$ is odd. However, a factor $p^{a}$ with $a$ a even number does not contribute with a factor 2 in the first summand of $m$.

It is easy to prove that, if $m$ is a perfect number, or $s(m)>m$, and we have $m$ a proper divisor of $n$, then $s(n)>n$ (of course, we are not considering the trivial case $n=$ perfect number). Thus, we have $\sigma(m) \geq 2 m$ and $n=l m$ with $l>1$. To prove the claim, we must check $\sigma(l m)>2 l m$. Let $\left\{1, d_{1}, d_{2}, \ldots, d_{r}\right\}$ be the divisors of $m$. Then, $1, l, l d_{1}, l d_{2}, \ldots, l d_{r}$ are divisors of $l m$. Therefore, $\sigma(l m) \geq 1+l+l d_{1}+l d_{2}+\cdots+l d_{r}>l\left(1+d_{1}+d_{2}+\cdots+d_{r}\right)=l \sigma(m) \geq 2 l m$.

As a consequence, all the drivers, with the exception of 2 (the "downdriver", as we show below), make $s(n)>n$. And, as the structure is preserved, it seems that we can get an always increasing sequence. But a driver can, eventually, disappear. Let us see again some examples; this time, with the driver $2^{3} \cdot 3$. Here, and in what follows, we will use $p, q$ to denote distinct prime numbers, being $p, q>3$.

When we have a number with the form $n=2^{3} \cdot 3 p$, it follows $s(n)=(1+2+4+$ 8) $\cdot 4(p+1)-2^{3} \cdot 3 p=2^{2} \cdot 3 \cdot[5(p+1)-2 p]$. Now, if $p$ has the form $p=4 r+1$, then $s(n)=2^{3} \cdot 3 \cdot[5(2 r+1)-p]$, and the expression between square brackets is even, so the power of 2 in $s(n)$ is at least 4 . However, if $p=4 r+3$, we get $s(n)=2^{3} \cdot 3 \cdot[5(2 r+2)-p]$ and, this time, the expression between square brackets is odd, so the power of 2 remains.

The driver $2^{3} \cdot 3$ can also disappear with numbers of the form $n=2^{3} \cdot 3^{2} p q$. Here, $s(n)=15 \cdot 13(p+1)(q+1)-8 \cdot 9 p q$. If $p, q \equiv 1 \bmod 4$, then the $2^{3}$ disappears and, 
in its place, $2^{2}$ arises. If $p, q \equiv 3 \bmod 4$, the $2^{3}$ persists. If $p \equiv 1$ and $q \equiv 3 \bmod 4$, then, at least, we have a factor $2^{3}$; but the power of 2 goes up if 8 is not a divisor of $q+1$, i.e., if $q \equiv 3 \bmod 8$.

When the driver of the sequence is 2 , the sequence usually decreases. For instance, if $n=2 p q$, then $s(n)=3(p+1)(q+1)-2 p q=p q+3(p+q+1)$. When $p$ and $q$ are big enough, $3(p+q+1)$ is tiny compared with $p q$, and so $s(n)<n$.

As for the other drivers, the driver 2 can disappear. For instance, let $n$ be $n=2 p$, with $s(n)=3(p+1)-2 p=p+3$. If $p=4 r+3$, then $s(n)=2(2 r+3)$. But, if $p=4 r+1$, then $s(n)=2^{2}(r+1)$ and so we have, at least, a factor $2^{2}$. The behavior is similar if $n=2 p^{2} q$, or if $n$ has more prime factors raised to even powers.

Guides in the form $2^{a}$ for $a>1$ make the sequence oscillate; we have $s(n)<n$ or $s(n)>n$ according to the other factors. And these guides change very easily.

And, what occurs when $n$ is odd? If $n=p$ prime, $s(n)=1$ and the sequence finishes. Now, let us assume $n=p q$. Then, $s(n)=(p+1)(q+1)-p q=p+q+1$ is also odd (usually, $s(n)<n$ ). The same occurs, for instance, with $n=p^{2} q$. But it is also possible that $n$ is odd but $s(n)$ is even. This is what happens when $n=p^{2}$; indeed, in this case $s(n)=\left(p^{2}+p+1\right)-p^{2}$, that is even.

It is possible (but not very common) that an aliquot sequences reaches a perfect number, an amicable pair or a cycle; this is the end of the aliquot sequence. Otherwise, to get a terminating sequence, it must reach an odd prime number. Usually, most terms of a sequence are even. Let us see the way in which a even term $n$ changes to an odd term $s(n)$. This occurs when $n=2^{a} p^{2}$, with $a>0$ (or similar cases with $p^{2 b}$ or more primes up to even powers). Indeed, $s(n)=\left(2^{a+1}-1\right)\left(1+p+p^{2}\right)-2^{a} p^{2}$, an odd number. Only chance can say whether this term is prime or not. Anyway, the sequence either reachs an even term (case previously considered) or it continues with odd tems until a prime term appears, where it stops.

Finally, let us also note that, althoug it seems unlikely that an always increasing aliquot sequence exists (of course, this would refute Catalan-Dickson conjecture), $\mathrm{H}$. W. Lenstra proved that it is possible to construct arbitrarily long monotonic increasing aliquot sequences (that is, for any $k$, we can found $m$ such that $s^{0}(m)<$ $\left.s^{1}(m)<\cdots<s^{k}(m)\right)$; the proof can be found in Erdös 76.

\section{BACKGROUND}

Many other people have been working with aliquot sequences, including P. Poulet, D. H. Lehmer, P. Erdős, J. Godwin, H. Cohen, R. K. Guy, M. Dickerman, H. J. J. te Riele, .... A extensive bibliography on this subject can be found in Guy 94, B6].

The first sequence whose end was in doubt started with 138; Lehmer found its end $s^{177}(138)=1$. For numbers $n<1000$, Lehmer could not find the end of the sequences starting from 276, 552, 564, 660, 840 and 966. Except for 840, which finished with $s^{747}(840)=1$ (found by A. Guy and R. K. Guy), the end for the others is still unknown; they are the "Lehmer five". In a similar way, the doubtful sequences starting between 1000 and 2000 are known as Godwin sequences; now, "Godwin twelve".

Regarding classifications of unknown sequences, let us note the following. Suppose that, for two different numbers $n_{1}$ and $n_{2}$ (say, $n_{1}<n_{2}$ ) there exist $k_{1}$ and $k_{2}$ such that $s^{k_{1}}\left(n_{1}\right)=s^{k_{2}}\left(n_{2}\right)$. Then, both sequences are the same from then on. In 
this case, we say that $n_{1}$ is the main sequence and $n_{2}$ is a side sequence. Only the main sequences are studied.

According to (1) or 22, to compute an aliquot sequence, we have to decompose $m$ into factors to calculate $s(m)$; and this, for many $m$ 's. This is hard when the number $m$ is large. The discovery of better factoring methods and the increase in the speed of computers lead to progress in the experimental knowledge of the behavior of aliquot sequences.

Recently, different people have extended the range of aliquot sequences that have been studied; W. Bosma, J. Gerved, S. Wagstaff, P.-L. Montgomery, H. J. J. te Riele, W. Lioen, A. Lenstra, and the authors have made contributions. Here are some recent advances.

In [Benito and Varona 99], two of the authors, following previous work of A. Guy and R. K. Guy, show a table with the status of doubtful main aliquot sequences starting at $n<10000$. In this paper we give an update: see Table 1 .

In the table, we include the number of decimal digits of the last term $s^{k}(n)$ known for each sequence and the guide in that stage. Actually, all the sequences have a driver in the current stage (a driver is more stable than a guide), except the ones marked with ${ }^{*}$.

In Benito and Varona 99, all the sequences in the table had been pursued up to, at least, 75 decimal digits. Now, we have reached more that 95 digits for all of them; and 100 digits for many of them. We have reached at least 112 digits for the Lehmer sequences and 101 digits for the Godwin sequences.

It is curious to note that the driver $2^{9} \cdot 3 \cdot 11 \cdot 31$ has appeared in no place in any of the sequences related in Table 1 .

W. Creyaufmüller is leading a project to study all the aliquot sequences starting at every $n \leq 10^{6}$; of course, there are many of them, so they are been studied up to less digits than in this paper. For a compilation of his work, see Creyaufmüller 00]. Here, we summarize the present state of such aliquot sequences with unknown status in Table 2,

We have not found new experimental evidences in favour of Guy-Selfridge conjecture, because drivers and guides disappear from time to time. For instance, when we increase the size of the last term reached for the sequences starting at every $n \leq 10^{6}$, the percentage of unknown sequences decreases in a significative way.

It seems feasible that Catalan's conjecture is true. But the question is, "How big are intermediate numbers that we need to factor to reach the end of the sequence?" As we see below, for $n=840$, we had to go up to 49 digit numbers; for $n=1248$, up to 58 digit numbers; for $n=3630$, up to 100 digit numbers. Perhaps we must go up to 200 or 1000 digit numbers for $n=276$, which would make this sequence out of reach. (Perhaps only 130 digits, which would make this sequence computable with today's computers and algorithms.)

Theoretically speaking, it does not seem an easy question. How many time will remain this problem open?

\section{Maximum of a terminating Sequence}

There has been considerable progress with respect to the height of aliquot sequences that are known to terminate. When D. H. Lehmer found the end of the aliquot sequence starting at 138 he found a maximum $s^{117}(138)$ of 12 decimal digits. Later, A. Guy and R. K. Guy found the end for 840 after finding a maximum 
TABLE 1. Aliquot sequences whose end is in doubt.

\begin{tabular}{|c|c|c|c|c|c|c|c|c|c|}
\hline$n$ & 276 & 552 & 564 & 660 & 966 & 1074 & 1134 & 1464 & 1476 \\
\hline$k$ & 1284 & 818 & 3048 & 468 & 526 & 1585 & 2249 & 1897 & 1055 \\
\hline digits & 117 & 118 & 115 & 112 & 114 & 105 & 127 & 101 & 106 \\
\hline guide & $2 \cdot 3$ & $2^{5} \cdot 3 \cdot 7$ & $2^{2} \cdot 7$ & $2^{2(*)}$ & $2 \cdot 3$ & $2^{2} \cdot 7$ & $2^{5} \cdot 3 \cdot 7$ & $2^{2} \cdot 7$ & $2^{3} \cdot 3 \cdot 5$ \\
\hline$n$ & 1488 & 1512 & 1560 & 1578 & 1632 & 1734 & 1920 & 1992 & 2232 \\
\hline$k$ & 824 & 1632 & 1336 & 1109 & 713 & 1404 & 1992 & 985 & 390 \\
\hline digits & 103 & 101 & 101 & 104 & 102 & 103 & 108 & 102 & 102 \\
\hline guide & $2^{2} \cdot 7$ & $2^{2} \cdot 7$ & $2^{5} \cdot 3 \cdot 7$ & $2^{2(*)}$ & $2^{3} \cdot 3 \cdot 5$ & $2^{2} \cdot 7$ & $2^{2} \cdot 7$ & $2^{3} \cdot 3 \cdot 5$ & $2^{6(*)}$ \\
\hline$n$ & 2340 & 2360 & 2484 & 2514 & 2664 & 2712 & 2982 & 3270 & 3366 \\
\hline$k$ & 471 & 974 & 796 & 2866 & 761 & 1347 & 826 & 417 & 1062 \\
\hline digits & 99 & 95 & 97 & 105 & 100 & 95 & 97 & 98 & 100 \\
\hline guide & $2^{3} \cdot 3 \cdot 5$ & $2^{2} \cdot 7$ & $2^{3} \cdot 3$ & $2^{3} \cdot 3 \cdot 5$ & $2^{2} \cdot 7$ & $2^{2} \cdot 7$ & $2^{4} \cdot 31$ & $2^{5} \cdot 3 \cdot 7$ & $2^{3(*)}$ \\
\hline$n$ & 3408 & 3432 & 3564 & 3678 & 3774 & 3876 & 3906 & 4116 & 4224 \\
\hline$k$ & 840 & 933 & 779 & 1201 & 1193 & 830 & 704 & 1192 & 519 \\
\hline digits & 95 & 103 & 100 & 98 & 98 & 96 & 100 & 105 & 98 \\
\hline guide & $2^{3} \cdot 3 \cdot 5$ & $2^{3} \cdot 3 \cdot 5$ & $2^{3} \cdot 3$ & $2^{2} \cdot 7$ & $2^{7} \cdot 3^{(*)}$ & $2^{2} \cdot 7$ & $2^{2} \cdot 7$ & $2^{3} \cdot 3$ & $2^{3} \cdot 3 \cdot 5$ \\
\hline$n$ & 4290 & 4350 & 4380 & 4788 & 4800 & 4842 & 5148 & 5208 & 5250 \\
\hline$k$ & 953 & 1165 & 965 & 2152 & 1135 & 473 & 1545 & 1710 & 1567 \\
\hline digits & 106 & 97 & 100 & 105 & 101 & 98 & 95 & 96 & 100 \\
\hline guide & $2^{2} \cdot 7$ & $2^{2} \cdot 7$ & $2^{2} \cdot 7$ & $2^{3} \cdot 3 \cdot 5$ & $2^{4} \cdot 31$ & $2^{2} \cdot 7$ & $2 \cdot 3$ & $2^{3} \cdot 3 \cdot 5$ & $2^{4(*)}$ \\
\hline$n$ & 5352 & 5400 & 5448 & 5736 & 5748 & 5778 & 6160 & 6396 & 6552 \\
\hline$k$ & 746 & 2776 & 1185 & 1093 & 1091 & 742 & 1630 & 1272 & 932 \\
\hline digits & 106 & 102 & 96 & 100 & 108 & 95 & 96 & 105 & 102 \\
\hline guide & $2^{2} \cdot 7$ & $2^{2} \cdot 7$ & $2^{3} \cdot 3 \cdot 5$ & $2^{2} \cdot 7$ & $2^{2} \cdot 7$ & $2^{4} \cdot 31$ & $2^{2(*)}$ & $2^{3} \cdot 3 \cdot 5$ & $2^{3} \cdot 3$ \\
\hline$n$ & 6680 & 6822 & 6832 & 6984 & 7044 & 7392 & 7560 & 7890 & 7920 \\
\hline$k$ & 1880 & 1177 & 885 & 1764 & 1113 & 498 & 846 & 891 & 1014 \\
\hline digits & 106 & 97 & 104 & 96 & 102 & 96 & 97 & 99 & 109 \\
\hline guide & $2^{4} \cdot 31$ & $2^{4} \cdot 31$ & $2^{3} \cdot 3$ & $2^{4} \cdot 31$ & $2^{4} \cdot 31$ & $2^{3} \cdot 3 \cdot 5$ & $2^{3} \cdot 5^{(*)}$ & $2^{2} \cdot 7$ & $2^{6} \cdot 127$ \\
\hline$n$ & 8040 & 8154 & 8184 & 8288 & 8352 & 8760 & 8844 & 8904 & 9120 \\
\hline$k$ & 2240 & 647 & 1241 & 849 & 1291 & 2157 & 1184 & 963 & 580 \\
\hline digits & 106 & 96 & 102 & 103 & 96 & 97 & 101 & 95 & 103 \\
\hline guide & $2^{3} \cdot 3 \cdot 5$ & $2^{2} \cdot 7$ & $2^{2} \cdot 7$ & $2^{8(*)}$ & $2^{2} \cdot 7$ & $2^{4(*)}$ & $2^{4} \cdot 31$ & $2^{2} \cdot 7$ & $2^{3} \cdot 3$ \\
\hline$n$ & 9282 & 9336 & 9378 & 9436 & 9462 & 9480 & 9588 & 9684 & 9708 \\
\hline$k$ & 556 & 608 & 2198 & 638 & 447 & 1028 & 1848 & 643 & 710 \\
\hline digits & 106 & 97 & 101 & 102 & 97 & 98 & 103 & 101 & 106 \\
\hline guide & $2^{3} \cdot 3$ & $2^{6} \cdot 127$ & $2^{2} \cdot 7$ & $2 \cdot 3$ & $2^{3} \cdot 3 \cdot 5$ & $2 \cdot 3$ & $2^{5} \cdot 3 \cdot 7$ & $2^{5} \cdot 3 \cdot 7$ & $2^{2} \cdot 7$ \\
\hline$n$ & 9852 & & & & & & & & \\
\hline$k$ & 669 & & & & & & & & \\
\hline digits & 105 & & & & & & & & \\
\hline guide & $2^{2} \cdot 7$ & & & & & & & & \\
\hline
\end{tabular}

$s^{287}(840)$ of 49 digits; and M. Dickerman did the same for 1248 , with a maximum $s^{583}(1248)$ of 58 digits. (Of course, if $p$ is a prime number, $s(p)=1$ so the sequence starting in $p$ finishes trivially. These trivial results are not considered in records.)

In Benito and Varona 99], we show that the sequence starting at 4170 converges to 1 after 869 iterations, getting a maximum of 84 decimal digits at iteration 289; this was a new record for the highest terminating aliquot sequence (found in December 22, 1996).

Later, in October 1999, W. Bosma broke this record: he found that the aliquot sequence starting with 44922 terminates after 1689 iterations (at 1), after reaching a maximum of 85 digits at step 1167. In December 3, 1999, he broke again the record finding that the sequence starting at 43230 finished: it terminates (at 1) after 4357 iterations, after reaching a maximum of 91 digits at step 967. 
TABLE 2. Number of aliquot sequences of unknown status starting with a number $\leq 10^{6}$.

\begin{tabular}{ccc}
\hline Interval & Number of sequences & Limits of computation \\
\hline$[1,100000]$ & 925 & $>10^{80}$ \\
$(100000,200000]$ & 975 & $>10^{80}$ \\
$(200000,300000]$ & 963 & $>10^{60}$ \\
$(300000,400000]$ & 903 & $>10^{60}$ \\
$(400000,500000]$ & 940 & $>10^{60}$ \\
$(500000,600000]$ & 990 & $>10^{60}$ \\
$(600000,700000]$ & 987 & $>10^{60}$ \\
$(700000,800000]$ & 990 & $>10^{80}$ \\
$(800000,900000]$ & 982 & $>10^{80}$ \\
$\left(900000,10^{6}\right]$ & 987 & \\
\hline
\end{tabular}

As of June 10, 2001, M. Benito and J. L Varona have found the end of the aliquot sequence starting at 3630 . It finishes at $s^{2624}(3630)=1$ after reaching a maximum $s^{1263}(3630)$ with a hundred digits, and is the present record for the highest known terminating aliquot sequence. In Figure 1 we show the shape of the sequence: in the horizontal axis, the index $k$ appears; and in the vertical axis, $\log _{10}\left(s^{k}(3630)\right)$.

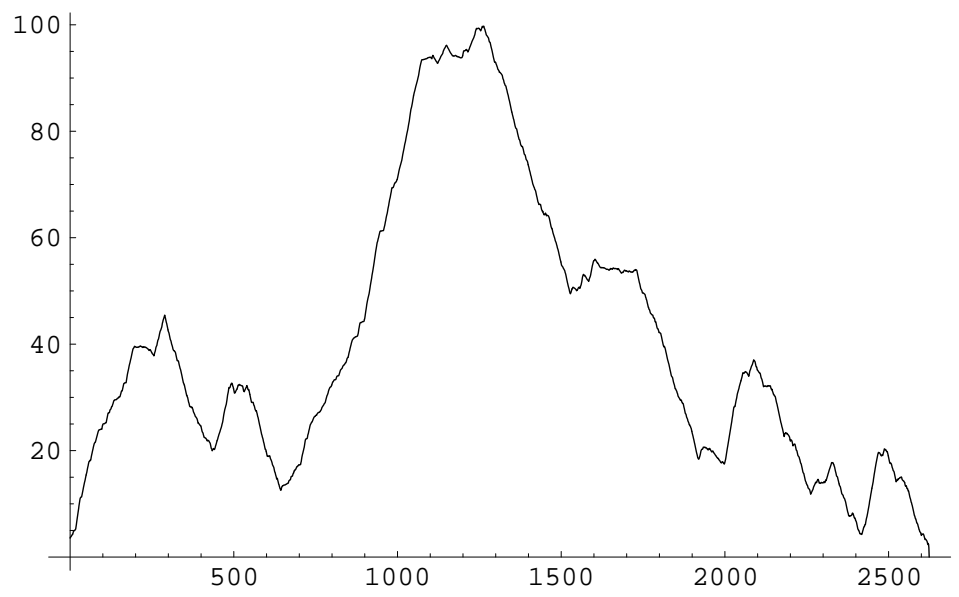

Figure 1. The aliquot sequence starting at 3630

Moreover, $3630<10000$ so the present Table 1 has one fewer entry than the table that appears in Benito and Varona 99. (the aforementioned sequences found by W. Bosma start in a number $>10000)$. Now, there are 82 main aliquot sequences (starting in a number $<10000$ ) whose end is still unknown.

For sequences of unknown status, the sequence 1134 has been extended by P. Zimmermann up to 127 digits; this is the largest term of an aliquot sequence computed so far. 
We maintain the following web pages related to aliquot sequences: http://home.t-online.de/home/Wolfgang.Creyaufmueller/aliquote.htm http://www.unirioja.es/dptos/dmc/jvarona/aliquot.html http://www.loria.fr/ zimmerma/records/aliquot.html

On these pages, one can find links to the aliquot web pages of other authors (in particular, from W. Bosma and J. Howell). The sequences of Table 1 can also be found at the $\mathrm{ftp}$ server

ftp://mat.unirioja.es/pub/aliquot/00xxxx

\section{THE METHOD}

As we already commented, to compute an aliquot sequence, we have to decompose $m$ into factors to calculate $s(m)$, and iterate this procedure a lot of times. This is hard when the number $m$ is large. And we are dealing with numbers up to more than a hundred of digits, so powerful algorithms are necessary. In particular, we have used the elliptic curve method (ECM) and the multiple polynomial quadratic sieve (MPQS); see a recent report (with many references) about the use of MPQS in [Boender and te Riele 96]. We have checked the primality of the factors with the Adleman-Pomerance-Rumely (APR) primality test.

All our work has been done by using packages available on the internet. The authors began to investigate the behavior of aliquot sequences around 1985. They have used many different kinds of computers, mainly PCs, but also Unix workstations and Macintoshes. In total, approximately 200 years of CPU time has been used.

The following packages, that are available at their corresponding web pages (or anonymous ftp sites):

- UBASIC, ftp://rkmath.rikkyo.ac.jp/pub/ubibm/

- PARI-GP, http://www.parigp-home.de/

- KANT-KASH (see Daberkow et al. 97]), http://www.math.tu-berlin. de/algebra/

- MIRACL, http://indigo.ie/ mscott/

- GMP, http://www.swox.com/gmp/

UBASIC programs to get aliquot sequences can be downloaded in Creyaufmüller's web page. PARI-GP and KANT-KASH programs can be downloaded in Varona's web page. A implementation of ECM for GMP can be downloaded in Zimmermann's web page.

One of the most titanic efforts undertaken has been to factorize the 109-digit cofactor in $s^{1267}(276)$. The computation time has been the equivalent to about 220 days of CPU time on an $800 \mathrm{Mhz}$ Pentium III computer (actually, the sieving has been carried out on a cluster of PCs). This kind of numbers is the reasonable limit for the MPQS method. For bigger ones, it will be better to use a new method of factorization: the number fields sieve (NFS); see, for instance Cavallar et al. 99.

\section{ACKNOWLEDGMENTS}

The authors thank all people who helped factorizing numbers from the Lehmer and several Godwin (1074 and 1134) sequences: Conrad Curry, Jim Howell, Arjen Lenstra, Paul Leyland, Sam Wagstaff. Whe also thank Wieb Bosma for his calculations on aliquot sequences starting with a number between 10000 and 50000 . 
Also, thanks to Peter Montgomery for making his program ecmfft available, Herman te Riele and Walter Lioen for their MPQS program, Sam Wagstaff for having done some aliquot factorizations on his MasPar, and Arjen Lenstra for his enormous help with the ppmpqs program. And to Yuji Kida for his programs (ECM and PPMPQS implementations) written in UBASIC.

Finally, thanks to Laureano Lambán, Eloy Mata, David Ortigosa and Julio Rubio for allowing us to use their personal computers to do some of the computations that appear in this paper.

The first author was on a study leave supported by Consejería de Educación, Cultura, Juventud y Deportes of Gobierno de La Rioja. Research of the third author was supported by Grant BFM2000-0206-C04-03 of the DGI and API-01/B38 of the UR.

\section{REFERENCES}

[Benito and Varona 99] M. Benito and J.L. Varona, Advances in aliquot sequences, Math. Comp. 68 (1999), 389-393.

[Boender and te Riele 96] H. Boender and H.J.J. te Riele, Factoring integers with large-prime variations of the quadratic sieve, Experimental Mathematics 5 (1996), 257-273.

[Cavallar et al. 99] S. Cavallar, B. Dodson, A.K. Lenstra, P.C. Leyland, W.M. Lioen, P.L. Montgomery, B.A. Murphy, H.J.J. te Riele, and P. Zimmermann, Factorization of RSA-140 using the Number Field Sieve, in Advances in Cryptology, Asiacrypt'99 (Berlin, 1999), Lecture Notes in Computer Science 1716, pp. 195-207, Springer-Verlag, Berlin-Heidelberg, 1999.

[Creyaufmüller 00] W. Creyaufmüller, Primzahlfamilien (3rd ed.), Verlagsbuchhandlung Creyaufmüller, Stuttgart, 2000.

[Daberkow et al. 97] M. Daberkow, C. Fieker, J. Klüners, M. Pohst, K. Roegner, M. Schörnig, and K. Wildanger, KANT V4, J. Symbolic Comput. 24 (1997), 267-283.

[Erdős 76] P. Erdős, On asymptotic properties of aliquot sequences, Math. Comp. 30 (1976), 641-645.

[Guy 94] R.K. Guy, Unsolved Problems in Number Theory (2nd ed.), Springer-Verlag, 1994.

[Guy and Selfridge 75] R.K. Guy and J.L. Selfridge, What drives an aliquot sequence?, Math. Comp. 29 (1975), 101-107. Corrigendum, ibid. 34 (1980), 319-321.

Instituto Sagasta, Glorieta del Doctor Zubía s/n, 26003 Logroño, Spain

E-mail address: mbenit8@palmera.pntic.mec.es

Freie Waldorfschule Aachen, Anton-Kurze-Allee 10, D-52074 Aachen, Germany

E-mail address: Wolfgang.Creyaufmueller@t-online.de

Departamento de Matemáticas y Computación, Universidad de la Rioja, Edificio J. L. Vives, Calle Luis de Ulloa s/n, 26004 Logroño, Spain

E-mail address: jvarona@dmc.unirioja.es

inRIa lorraine, Technopôle de Nancy-Brabois, 615 rue du Jardin Botanique, BP 101, F-54600 Villers-LÈS-NANCY, FrANCE

E-mail address: Paul.Zimmermann@inria.fr 